\title{
Ultrasound-guided percutaneous portal transplan- tation of peripheral blood monocytes in patients with liver cirrhosis
}

\author{
Su Jong $\mathrm{Yu}^{1}$, Jung-Hwan Yoon ${ }^{1}$, Won $\mathrm{Kim}^{2}$, Jeong Min Lee³ , Yun Bin Lee ${ }^{1}$, Yuri Cho ${ }^{1}$, Dong Hyeon Lee

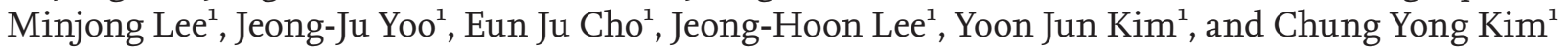

\begin{abstract}
${ }^{1}$ Department of Internal Medicine and Liver Research Institute, Seoul National University College of Medicine, Seoul; ${ }^{2}$ Department of Internal Medicine, Seoul Metropolitan Government Seoul National University Boramae Medical Center, Seoul; ${ }^{3}$ Department of Radiology and Institute of Radiation Medicine, Seoul National University College of Medicine, Seoul, Korea
\end{abstract}

Received: August 11, 2015

Revised : September 2, 2015

Accepted: September 6, 2015

Correspondence to

Jung-Hwan Yoon, M.D.

Department of Internal

Medicine and Liver Research

Institute, Seoul National

University College of Medicine,

101 Daehak-ro, Jongno-gu, Seoul

03080, Korea

Tel: $+82-2-2072-2228$

Fax: +82-2-743-6701

E-mail:yoonjh@snu.ac.kr
Background/Aims: Liver transplantation offers the only definite cure for cirrhosis but lacking donors is problem. Stem cell therapy is attractive in this setting. In this study, we aimed to explore the safety and efficacy of ultrasound-guided percutaneous portal transplantation of peripheral blood monocyte cell (PBMC) in cirrhotic patients.

Methods: A total of nine decompensated cirrhotic patients were randomized into three groups: group $1(n=3)$ was control group, group $2(n=3)$ received granulocyte-colony stimulating factor (G-CSF) mobilization for 3 days, and group $3(\mathrm{n}=3)$ received G-CSF mobilized PBMCs by leukapheresis and PBMC transplantation through ultrasound-guided percutaneous portal vein puncture. Liver function and clinical features were evaluated.

Results: At baseline, the Child-Turcotte-Pugh and the model for end-stage liver disease scores were comparable in study groups. Compared with group 1, there was a tendency to improve liver function in group 3 at 6 months after treatment. Treatment was tolerable and no complications were encountered related to the G-CSF mobilization or percutaneous portal administration of PBMCs. Imaging studies showed patent portal veins at the end of the study period.

Conclusions: Autologous PBMC transplantation through ultrasound-guided percutaneous portal vein puncture could be considered as a safe alternative treatment for decompensated cirrhotic patients.

Keywords: Leukocytes, mononuclear; Granulocyte colony-stimulating factor; Liver cirrhosis

\section{INTRODUCTION}

Liver cirrhosis is an irreversible result of advanced liver disease and treatment usually focuses on preventing progression and complications. Although liver transplantation (LT) provides the only curative treatment [1], LT is restricted by lack of donor organs, operative damage, and risk of rejection and high cost of the procedure
[2]. Therefore, an alternative therapy to improve the liver function of cirrhotic patients with minimally invasive procedures is urgently needed.

Adult stem cell-based regenerative therapies are evolving as viable clinical alternatives. There have been several reports that bone marrow stem cells (BMSCs) could accelerate the recovery and improvement of liver function in animal models of liver injury [3-5]. The potential of BM- 
SCs to differentiate into human hepatocytes had been identified in liver biopsies after sex-mismatched orthotopic LT $[6,7]$. The intriguing capability of blood-derived stem cells that differentiate into multiple cell lineages raises the exciting opportunity of using these cells for tissue repair [8]. In humans, Alison et al. [7] and Theise et al. [6] demonstrated that hepatocytes could also be derived from bone marrow cell populations, although the degree of hepatic engraftment of stem cells into the human liver appeared to be highly variable.

The clinical experience in using adult stem cells for the treatment of liver damage is limited. Only a few clinical studies have been reported in which BMSCs or granulocyte-colony stimulating factor (G-CSF)-mobilized peripheral blood hematopoietic stem cells could contribute to the liver function [9-12]. A previous study showed that peripheral blood monocyte cells (PBMCs) from patients with hepatitis B virus-related decompensated liver cirrhosis could differentiate into functional hepatocytes and contribute to liver function [13]. Although the preliminary results seemed attractive, there has been no consensus in the source of adult stem cells and the route of cells transfer to evaluate the safety and efficacy of stem cell therapy in cirrhotic patients. In the light of these findings, we conducted a prospective clinical study of G-CSF-mobilized PBMC transplantation through ultrasound-guided percutaneous portal vein puncture in cirrhotic patients to determine whether clinical safety and benefit was conferred.

\section{METHODS}

\section{Patients}

From July 2012 to January 2013, 14 cirrhotic patients were screened from affiliated hospitals of Seoul National University Hospital. Inclusion criteria were as follows: age 20 to 79 years, decompensated liver cirrhosis with Child-Turcotte-Pugh (CTP) score 8 or 9 (including patients who have no radiologic evidence of remnant hepatocellular carcinoma [HCC] for more than 2 years after treatment). Exclusion criteria were as follows: age less than 20 or more than 80 years; serum hepatitis B surface antigen positive; who had not abstained from alcohol for at least 6 months; active status of HCC (except patients who have no radiologic evidence of remnant HCC for more than 2 years after treatment); history of hemochromatosis and/or autoimmune hepatitis; pregnant women or lactating women; hemoglobin $<8 \mathrm{~g} / \mathrm{dL}$ (male)/7.5 g/dL (female) or white blood cell $<1,500 \mathrm{~mm}^{3}$ or neutrophils $<500 / \mathrm{mm}^{3}$ or platelet count $<50,000 / \mathrm{mm}^{3}$; serum creatinine $>1.5 \times$ normal upper limit or creatinine clearance $<60 \mathrm{~mL} / \mathrm{min}$; presence of signs of malignant tumors or tumor suspected symptoms or history of malignant tumors with recurrence rate greater than $20 \%$ within 2 years; gastrointestinal bleeding within the last 3 months or if there is a history of spontaneous bacterial peritonitis; presence of portal vein thrombosis; presence of acute infections. The total number of enrolled subjects consisted of nine patients. This study was reviewed and approved by the Institutional Review Board of Seoul National University Hospital (IRB No. 1110-126-383) and a written informed consent was obtained from each patient (NCT01503749).

\section{G-CSF mobilization and PBMC transplantation therapy}

The patients were randomized into three groups: (1) group 1 (control) did not receive any intervention regarding to PBMCs; (2) group 2 received G-CSF mobilization at a dose of $5 \mu \mathrm{g} / \mathrm{kg} /$ day subcutaneously for 3 days; and (3) group 3 received PBMCs transplant therapy. A random number table was used for simple randomization of subjects [14]. PBMCs were mobilized with G-CSF at a dose of $5 \mu \mathrm{g} / \mathrm{kg} /$ day, administered subcutaneously daily for 3 days to induce the bone marrow derived PBMCs stem cells into the peripheral blood. On the day 4 th, PBMCs were collected by means of apheresis using COBE SPECTRA Apheresis System (Terumo BCT Ltd., Hong Kong). The duration of the procedure up to 3 hours until the number of PBMC reached to $1.67 \times 10^{9}-2 \times 10^{10} / 50$ $\mathrm{mL}$. Then $1.67 \times 10^{9}-2 \times 10^{10} / 50 \mathrm{~mL}$ PBMC were administered centrally to the patient at an infusion rate of 10 $\mathrm{mL} / \mathrm{min}$ through the ultrasound-guided percutaneous right portal branch puncture using 21 gauge TSK SONORI ject access needle (TSK Laboratory, Tochigi, Japan). Patients were discharged after 24 hours of bed rest.

\section{Follow-up}

Recruitment started in July 2012. The first patient in group 1 was recruited in August 2012, the first patient in group 2 in August 2012, and the first patient in group 
3 in July 2012. The CTP score and model for end-stage liver disease (MELD) score were calculated to assess the overall condition of the patients with decompensated liver cirrhosis at baseline and at weeks 2, 4, 8, 12, 16, 20, and 24. After treatment, all patients were followed-up for 6 months. Patients abstained from alcohol and did not receive antiviral therapies, such as pegylated interferon and ribavirin during follow-up period. Albumin was replaced only when paracentesis was performed. At baseline and after 6 months follow-up, abdominal imaging (ultrasonography or computed tomography) was performed for evaluation of patency of portal vein, ascites or surveillance of HCC.

\section{Statistical analysis}

Changes in laboratory data from baseline to 6 months after treatment among study groups were analyzed. All data are expressed as median and range, unless otherwise indicated. The comparative liver functions of the three groups were investigated by analysis of the Wilcoxon's signed ranks test (group 1 vs. 2 and group 1 vs. 3). Statistical analysis was done using SPSS version 19.0 (IBM Co., Armonk, NY, USA) and $p$ values $<0.05$ were considered statistically significant.

\section{RESULTS}

\section{Demographic and clinical characteristics of the patients}

Nine patients who fulfilled the inclusion and exclusion criteria were included in the study. The clinical profile was similar across the study groups (Table 1 ). There were two male patients in group 1 (male/female, 2:1), one male patients in group 2 (male/female, 1:2), and two male patients in group 3 (male/female, $2: 1 ; p=1.000$ ). The median age (61 years [range, 45 to 65] in group 1, 59 years [range, 59 to 68 ] in group 2, and 65 years [range, 54 to 71 ] in group $3 ; p=0.646$ ) was comparable in three groups. Five patients $(55.6 \%)$ had hepatitis C virus-related liver cirrhosis and alcoholic liver cirrhosis was seen in four patients (44.4\%). Puncture site of portal vein and injected cell number in each patient were summarized in Table 2.

\section{Changes in laboratory data after treatment}

At baseline, biochemical markers including total bilirubin, albumin, prothrombin time, creatinine, aspartate aminotransferase, alanine aminotransferase, the CTP scores, and the MELD scores were comparable in study groups. PBMC transplantation therapy showed a tendency to improve in median CTP scores at weeks 24

Table 1. Demographic and clinical characteristics of patients

\begin{tabular}{|c|c|c|c|c|c|c|c|c|c|}
\hline \multirow{2}{*}{ Characteristic } & \multicolumn{3}{|c|}{ Group $1(n=3)$} & \multicolumn{3}{|c|}{ Group $2(\mathrm{n}=3)$} & \multicolumn{3}{|c|}{ Group $3(n=3)$} \\
\hline & Patient 1 & Patient 2 & Patient 3 & Patient 4 & Patient 5 & Patient 6 & Patient 7 & Patient 8 & Patient 9 \\
\hline Age $(y r) /$ sex & $65 / \mathrm{M}$ & 61/M & $45 / \mathrm{F}$ & $59 / \mathrm{F}$ & $68 / \mathrm{F}$ & $59 / \mathrm{M}$ & $65 / \mathrm{M}$ & $54 / \mathrm{M}$ & $71 / F$ \\
\hline $\mathrm{BMI}, \mathrm{kg} / \mathrm{m}^{2}$ & 28.64 & 28.76 & $15 \cdot 35$ & 18.78 & 25.81 & 20.52 & 19.97 & 26.54 & 24.43 \\
\hline Etiologies & $\mathrm{HCV}$ & Alcohol & Alcohol & $\mathrm{HCV}$ & $\mathrm{HCV}$ & Alcohol & $\mathrm{HCV}$ & Alcohol & $\mathrm{HCV}$ \\
\hline HCV titer, IU/mL & 474,169 & NA & NA & 48,771 & 291,691 & NA & 236,758 & NA & 841,060 \\
\hline Ascites & Yes & No & Yes & Yes & No & Yes & Yes & Yes & Yes \\
\hline Encephalopathy & No & No & No & No & No & No & No & No & No \\
\hline Total bilirubin, mg/dL & 2.2 & $4 \cdot 5$ & $5 \cdot 4$ & 6.3 & $3 \cdot 4$ & 7.2 & $3 \cdot 9$ & $3 \cdot 5$ & 2.9 \\
\hline Albumin, g/dL & 3.2 & 2.9 & $3 \cdot 4$ & 2.8 & 2.4 & 2.8 & $3 \cdot 3$ & 3.2 & $3 \cdot 4$ \\
\hline Prothrombin time, INR & 1.14 & 1.91 & 1.26 & 1.66 & 1.62 & 1.68 & 1.12 & 1.67 & 1.3 \\
\hline Creatinine, mg/dL & 1.26 & 0.59 & 0.85 & 0.86 & 0.66 & 0.7 & 0.9 & 0.73 & 0.6 \\
\hline MELD score & 13.1 & 19.4 & $15 \cdot 4$ & 19.1 & 16.5 & 19.7 & 12.8 & 16.9 & 13 \\
\hline CTP score & 8 & 9 & 9 & 9 & 9 & 9 & 9 & 9 & 8 \\
\hline Diabetes & Yes & None & None & None & None & Yes & Yes & None & None \\
\hline
\end{tabular}

BMI, body mass index; HCV, hepatitis C virus; NA, not available; INR, international normalized ratio; MELD, model for endstage liver disease; CTP, Child-Turcotte-Pugh. 
compared to baseline (Fig. 1). However, there were no significant reductions in the median CTP and MELD scores among study groups probably due to small sample size (Fig. 2). In addition, there were no statistical differences in the median CTP and MELD scores among different etiologies of chronic liver diseases.

\section{Clinical outcome of the participants}

During the 6 months of follow-up, there was no mortality in study groups. None of patients in the study groups experienced variceal bleeding, hepatorenal syndrome, or spontaneous bacterial peritonitis. In group 1, one patient had a hepatic encephalopathy 2 months after enrollment (treated with lactulose enema). None of patients in group 2 and group 3 experienced hepatic encephalopathy. Three patients underwent paracentesis with albumin replacement (dosage of $20 \mathrm{~g} /$ each session): one patient at week 16 and 24 in group 1, one patient at week 20 in group 2, and one patient at week 12 (with thoracentesis) in group 3. HCC was not developed in all study patients during the follow-up period.

\section{Adverse events}

G-CSF therapy was very well tolerated in our patient
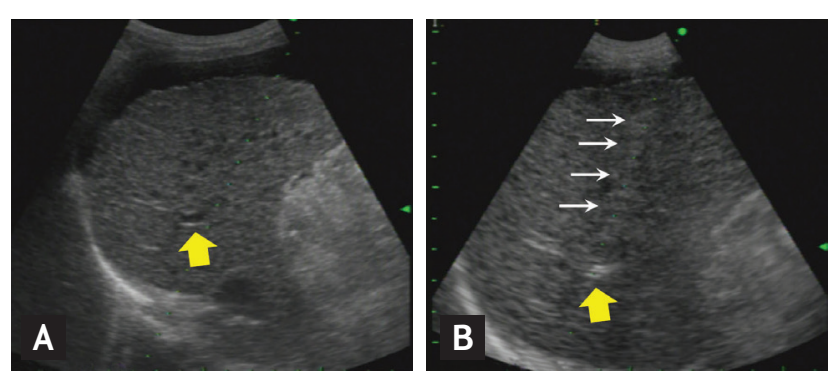

Figure 1. The procedure of ultrasound-guided percutaneous portal transplantation of peripheral blood monocyte cells (PBMCs). (A) Right portal vein (yellow arrows) was visualized by ultrasound. (B) After subcutaneous and subcapsular anesthesia by lidocaine, puncture needle (white arrows) was introduced into the portal vein through liver parenchyma while patient held breath for 10 seconds. Hyperechoic flow was observed in the portal vein during infusion of PBMCs through the puncture needle. group. None of the patients received G-CSF mobilization had splenic rupture during the study period. In addition, there was no bleeding or infection after portal administration of PBMCs. All of patients in group 3, imaging studies showed patent portal veins at the end of 6 months follow-up period. There were no significant adverse events related with G-CSF mobilization or PBMC transplantation during follow-up. Complications of the patients in group 1 were as follows: one patient had gingival bleeding, abdominal pain, and diarrhea. Complications of the patients in group 2 were as follows: one patient had abdominal pain, nausea, diarrhea, and hyperkalemia (treated with kalimate). There was no complication in group 3 .

\section{DISCUSSION}

This is the first study to present detailed transplantation procedures of autologous PBMCs to cirrhotic patients through the direct percutaneous needle puncture of portal vein under guidance of ultrasound. In this prospective controlled study, we have demonstrated the safety and potential of PBMC transplantation therapy for cirrhotic patients.

Intraportal administration of stem cells has been tried in four previous studies. In the first study, the portal catheter was introduced by minilaparotomy under general anesthesia and $\mathrm{CD} 133^{+}$bone marrow cells were administered through the portal catheter to three cirrhotic patients [11]. In the second study, autologous $\mathrm{CD}_{34}{ }^{+}$ cells were injected into the portal vein under computer tomography (CT) scan [12]. In the third study, a portal venogram was performed by percutaneous liver puncture under ultrasound guidance but infusion of $\mathrm{CD}_{34}{ }^{+}$ cells was performed via angiographic catheter under C-arm CT monitoring [15]. In the fourth study, the authors just stated that they delivered stem cells via one of the main branches of the portal vein under ultrasound guidance but they did not provide a well-described pro-

Table 2. PBMC cell numbers and injection routes

\begin{tabular}{lccc}
\hline & Patient 7 & Patient 8 & Patient 9 \\
\hline Injected cell number of PBMCs & $1.67 \times 10^{9}$ & $1.18 \times 10^{10}$ & $2.0 \times 10^{10}$ \\
Route of infusion & Right portal vein & Right portal vein & Right portal vein \\
\hline
\end{tabular}

PBMCs, peripheral blood monocyte cell. 


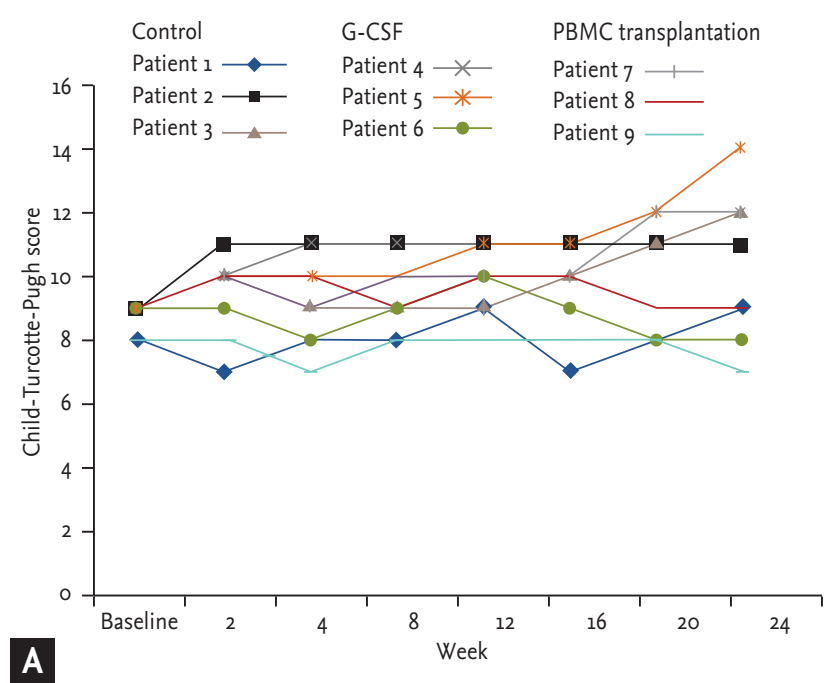

A

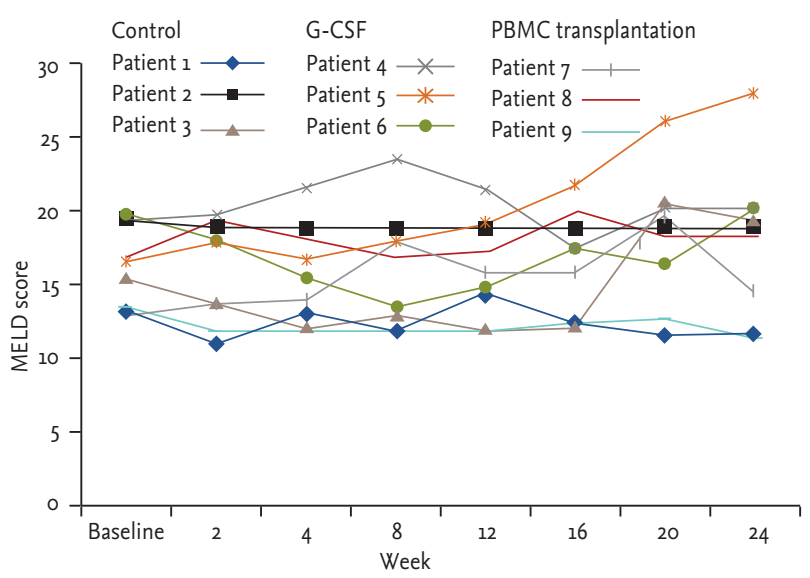

B

Figure 2. Post-transplantation follow-up of patients. (A) Changes in Child-Turcotte-Pugh score. (B) Changes in model for end-stage liver disease (MELD) score. G-CSF, granulocyte-colony stimulating factor; PBMC, peripheral blood monocyte cell.

tocol of portal application [16]. Therefore, this study had several superiorities over previous studies, including transient minimal invasiveness from percutaneous portal vein needle puncture, avoidance of radiation hazards from CT scan by using ultrasound-guidance, and providing a detailed protocol of portal application. In addition, it is important to note that direct portal application may have eased to some extent the homing mechanisms, physiologically required to attract circulating extrahepatic stem cells to the regenerating liver. Moreover, after the PBMCs were re-infused into the portal vein through the ultrasound-guided direct percutaneous needle puncture in this study, no bleeding or thrombotic episode was observed. Therefore, ultrasound-guided percutaneous portal transplantation of autologous PBMCs is assumed to be a well-tolerated therapy for cirrhotic patients.

Different types of stem cells are theoretically eligible for liver regeneration. These include fetal annex (placenta and cord blood) and embryonic stem cells $[17,18]$, induced pluripotent stem cells [19], hepatoblasts, and adult stem cells including hepatic progenitor cells, unsorted mononuclear stem cells, hematopoietic stem cells, and mesenchymal stem cells [20-22]. However, there have been no large randomized, controlled clinical trials comparing the efficacy of different types of stem cells. Currently, the most promising source for stem cell-based therapies is represented by the infusion of hematopoietic stem cells [23]. In this regard, we adopted PBMCs (traceable using CD34 and CD133 markers) as source of stem cells obtained from peripheral collection through leukapheresis after G-CSF administration which requires no additional invasive bone marrow aspiration and/or ex vivo manipulation $[20,24]$. G-CSF alone has been also tried in liver regeneration because of its ability to increase the number of circulating BMSCs, to enhance mononuclear stem cells homing to the liver, and to promote healing in the cirrhotic liver [25,26]. Although G-CSF administration can be also associated with the risk of spleen enlargement or even rupture in a healthy donor [27,28], Lorenzini et al. [29] demonstrated the safety of BMSC mobilization and collection through leukapheresis in cirrhotic patients. G-CSF administration was also safe and induced no serious adverse events in this study, but there was no significant clinical improvement over control group. The result of this study was consistent with previous studies evaluating G-CSF treatment, which demonstrated no clinical benefit [21,3032].

Although the mechanisms involved in stem cell therapy are not yet fully understood, some theories have been proposed [33,34]. One is that genomic plasticity evokes the trans-differentiation of stem cells into functioning hepatocytes in response to the microenvironment [35]. Another mechanism is presumably related to the cell fusion of BMSCs and hepatocytes [36]. It is crucial to understand the homing processes of stem cells to the liver and to elucidate the relationships that exist not only between stem cells and hepatocytes (regeneration) but 
also between mesenchymal stem cells, myofibroblasts and stellate cells (fibrogenesis). In addition, it has been suggested that stem cells may act through the delivery of specific substances (cytokines and growth factors), rather than through trans-differentiation or cell fusion, suggests that improvements in liver function might be temporary [37,38]. This hypothesis is supported by the results of the majority of the clinical trials: the improvement in laboratory data and CTP and MELD scores did not persist longer than 3 to 6 months regardless of the type of BMSCs infused, the route of delivery or the etiology of the disease. In addition, the histological evaluations support this hypothesis, as an increase in liver progenitor cells count was documented, peaking 3 months after stem cells infusion. According to the meta-analyses, stem cell therapy could improve the liver function without any severe procedure-related complications, but this improvement was insignificant compared with conventional treatment and not sustained at the 12th postoperative months [39]. Although this study was a pilot study and too small to make any comment regarding efficacy, autologous PBMCs administration showed only minimal significant clinical improvement over control group in our study. These results suggest that repeated cycles of stem cell therapy could be useful to obtain a sustained significant clinical benefit.

Ultrasound-guided percutaneous portal transplantation of autologous PBMCs to cirrhotic patients, although promising, needs to be further evaluated in large randomized, controlled clinical trials with repeated cycles of therapy. A better understanding of the action of stem cells in the context of a fibrotic liver might allow more rational use of autologous PBMC therapy in liver cirrhosis.

\section{KEY MESSAGE}

1. This is the first study to present detailed transplantation procedures of autologous peripheral blood monocyte cells (PBMCs) to cirrhotics through the direct percutaneous needle puncture of portal vein under guidance of ultrasound.

2. Direct portal transplantation of PBMCs for cirrhotic patients is a safe therapeutic modality.

\section{Conflict of interest}

No potential conflict of interest relevant to this article was reported.

\section{Acknowledgments}

This research was supported by Basic Science Research Program through the National Research Foundation of Korea (NRF) funded by the Ministry of Education, Science and Technology (Grant No. 20100007381) and by the Liver Research Foundation of Korea. We thank MRCC (medical research collaborating center) of Seoul National University Hospital for assistance in statistical analysis.

\section{REFERENCES}

1. Shim JH, Lee HC, Kim KM, et al. Efficacy of entecavir in treatment-naive patients with hepatitis B virus-related decompensated cirrhosis. J Hepatol 2010;52:176-182.

2. Han Y, Yan L, Han G, et al. Controlled trials in hepatitis $B$ virus-related decompensate liver cirrhosis: peripheral blood monocyte transplant versus granulocyte-colony-stimulating factor mobilization therapy. Cytotherapy 2008;10:390-396.

3. Pai M, Zacharoulis D, Milicevic MN, et al. Autologous infusion of expanded mobilized adult bone marrow-derived $\mathrm{CD}_{34}+$ cells into patients with alcoholic liver cirrhosis. Am J Gastroenterol 2008;103:1952-1958.

4. Lagasse E, Connors H, Al-Dhalimy M, et al. Purified hematopoietic stem cells can differentiate into hepatocytes in vivo. Nat Med 2000;6:1229-1234.

5. Korbling M, Katz RL, Khanna A, et al. Hepatocytes and epithelial cells of donor origin in recipients of peripheral-blood stem cells. N Engl J Med 2002;346:738-746.

6. Theise ND, Nimmakayalu M, Gardner R, et al. Liver from bone marrow in humans. Hepatology 2000;32:11-16.

7. Alison MR, Poulsom R, Jeffery R, et al. Hepatocytes from non-hepatic adult stem cells. Nature 2000;406:257.

8. Grove JE, Bruscia E, Krause DS. Plasticity of bone marrow-derived stem cells. Stem Cells 2004;22:487-500.

9. Terai S, Ishikawa T, Omori K, et al. Improved liver function in patients with liver cirrhosis after autologous bone marrow cell infusion therapy. Stem Cells 2006;24:22922298.

10. Yannaki E, Anagnostopoulos A, Kapetanos D, et al. Last- 
ing amelioration in the clinical course of decompensated alcoholic cirrhosis with boost infusions of mobilized peripheral blood stem cells. Exp Hematol 2006;34:1583-1587.

11. am Esch JS 2nd, Knoefel WT, Klein M, et al. Portal application of autologous CD133+ bone marrow cells to the liver: a novel concept to support hepatic regeneration. Stem Cells 2005;23:463-470.

12. Gordon MY, Levicar N, Pai M, et al. Characterization and clinical application of human $\mathrm{CD}_{34}+$ stem/progenitor cell populations mobilized into the blood by granulocyte colony-stimulating factor. Stem Cells 2006;24:1822-1830.

13. Yan L, Han Y, Wang J, Liu J, Hong L, Fan D. Peripheral blood monocytes from patients with HBV related decompensated liver cirrhosis can differentiate into functional hepatocytes. Am J Hematol 2007;82:949-954.

14. Suresh K. An overview of randomization techniques: an unbiased assessment of outcome in clinical research. J Hum Reprod Sci 2011;4:8-11.

15. Levicar N, Pai M, Habib NA, et al. Long-term clinical results of autologous infusion of mobilized adult bone marrow derived $\mathrm{CD}_{34}+$ cells in patients with chronic liver disease. Cell Prolif 2008;41 Suppl 1:115-125.

16. Kharaziha P, Hellstrom PM, Noorinayer B, et al. Improvement of liver function in liver cirrhosis patients after autologous mesenchymal stem cell injection: a phase I-II clinical trial. Eur J Gastroenterol Hepatol 2009;21:11991205.

17. Campard D, Lysy PA, Najimi M, Sokal EM. Native umbilical cord matrix stem cells express hepatic markers and differentiate into hepatocyte-like cells. Gastroenterology 2008;134:833-848.

18. Teramoto K, Asahina K, Kumashiro Y, et al. Hepatocyte differentiation from embryonic stem cells and umbilical cord blood cells. J Hepatobiliary Pancreat Surg 2005;12:196-202.

19. Piscaglia AC, Campanale M, Gasbarrini A, Gasbarrini G. Stem cell-based therapies for liver diseases: state of the art and new perspectives. Stem Cells Int 2010;2010:259461.

20. Margini C, Vukotic R, Brodosi L, Bernardi M, Andreone P. Bone marrow derived stem cells for the treatment of endstage liver disease. World J Gastroenterol 2014;20:90989105 .

21. Lorenzini S, Gitto S, Grandini E, Andreone P, Bernardi M. Stem cells for end stage liver disease: how far have we got? World J Gastroenterol 2008;14:4593-4599.

22. Fausto N. Liver regeneration and repair: hepatocytes, progenitor cells, and stem cells. Hepatology 2004;39:14771487.

23. Mallet VO, Gilgenkrantz H. Mobilizing stem cells to repair liver after surgery: dream or reality? J Hepatol 2005;43:754-756.

24. de Wynter EA, Buck D, Hart C, et al. CD34+AC133+ cells isolated from cord blood are highly enriched in longterm culture-initiating cells, NOD/SCID-repopulating cells and dendritic cell progenitors. Stem Cells 1998;16:387-396.

25. Mizunaga Y, Terai S, Yamamoto N, et al. Granulocyte colony-stimulating factor and interleukin-1 $\beta$ are important cytokines in repair of the cirrhotic liver after bone marrow cell infusion: comparison of humans and model mice. Cell Transplant 2012;21:2363-2375.

26. Jin SZ, Meng XW, Sun X, et al. Granulocyte colony-stimulating factor enhances bone marrow mononuclear cell homing to the liver in a mouse model of acute hepatic injury. Dig Dis Sci 2010;55:2805-2813.

27. Picardi M, De Rosa G, Selleri C, et al. Spleen enlargement following recombinant human granulocyte colony-stimulating factor administration for peripheral blood stem cell mobilization. Haematologica 2003;88:794-800.

28. Falzetti F, Aversa F, Minelli O, Tabilio A. Spontaneous rupture of spleen during peripheral blood stem-cell mobilisation in a healthy donor. Lancet 1999;353:555.

29. Lorenzini S, Isidori A, Catani L, et al. Stem cell mobilization and collection in patients with liver cirrhosis. Aliment Pharmacol Ther 2008;27:932-939.

30. Gaia S, Smedile A, Omede P, et al. Feasibility and safety of G-CSF administration to induce bone marrow-derived cells mobilization in patients with end stage liver disease. J Hepatol 2006;45:13-19.

31. Di Campli C, Zocco MA, Saulnier N, et al. Safety and efficacy profile of G-CSF therapy in patients with acute on chronic liver failure. Dig Liver Dis 2007;39:1071-1076.

32. Spahr L, Lambert JF, Rubbia-Brandt L, et al. Granulocyte-colony stimulating factor induces proliferation of hepatic progenitors in alcoholic steatohepatitis: a randomized trial. Hepatology 2008;48:221-229.

33. Kisseleva T, Brenner DA. The phenotypic fate and functional role for bone marrow-derived stem cells in liver fibrosis. J Hepatol 2012;56:965-972.

34. Lorenzini S, Andreone P. Regenerative medicine and liver injury: what role for bone marrow derived stem cells? Curr Stem Cell Res Ther 2007;2:83-88. 
35. Jang YY, Collector MI, Baylin SB, Diehl AM, Sharkis SJ. Hematopoietic stem cells convert into liver cells within days without fusion. Nat Cell Biol 2004;6:532-539.

36. Wang $X$, Willenbring $H$, Akkari $Y$, et al. Cell fusion is the principal source of bone-marrow-derived hepatocytes. Nature 2003;422:897-901.

37. Sakaida I, Terai S, Yamamoto N, et al. Transplantation of bone marrow cells reduces $\mathrm{CCl}_{4}$-induced liver fibrosis in mice. Hepatology 2004;40:1304-1311.
38. Wang L, Wang X, Wang L, et al. Hepatic vascular endothelial growth factor regulates recruitment of rat liver sinusoidal endothelial cell progenitor cells. Gastroenterology 2012;143:1555-1563.e2.

39. Qi X, Guo X, Su C. Clinical outcomes of the transplantation of stem cells from various human tissue sources in the management of liver cirrhosis: a systematic review and meta-analysis. Curr Stem Cell Res Ther 2015;10:166180 . 